\section{Revista Facultad 2020 \\ de Ciencias Básicas Vol. 16(2) \\ julio - diciembre}

\title{
Un nuevo estudio de los conjuntos Supra Neutrosophic Crisp*
}

\section{Carlos Granados}

Resumen: dado que el mundo está lleno de indeterminación, los Neutrosophic encontraron su lugar en la investigación contemporánea. Es por esto que en este artículo presentamos un nuevo resultado de conjuntos supra Neutrosophic Crisp sobre un espacio topológico supra Neutrosophic Crisp. En esta investigación se utilizó la noción de conjuntos supra Neutrosophic Crisp semi-a-cerrados para introducir nuevos conceptos, tales como propiedades de separación y nuevas nociones de conjuntos. Además, se encuentran algunas relaciones existentes entre ellas.

Palabras clave: teoría de conjuntos Neutrosophic; conjuntos supra Neutrosophic Crisp

semi-a-cerrados; Neutrosophic axiomas de separación; espacio topológico supra Neutrosophic Crisp;

espacio topológico

Recibido: 29 de octubre de 2020

Aceptado: 12 de enero de 2021

Disponible en línea: 27 de agosto de 2021

Cómo citar: C. Granados, «Un nuevo estudio de los conjuntos Supra Neutrosophic Crisp», Rev. Fac. Cienc. Básicas, vol. 16, n. ${ }^{\circ}$ 2, pp. 65-75, ago. 2021.

* Artículo de investigación.

a Magíster en Ciencias Matemáticas, Universidad del Atlántico, Barranquilla, Colombia. Correo electrónico: carlosgranadosortiz@outlook.es ORCID: https://orcid.org/0000-0002-7754-1468 


\section{A New Study of Supra Neutrosophic Crisp Sets}

Summary: since the world is full of indeterminacy, the Neutrosophic found their place in contemporary research. This is why in this article we present a new result of Supra Neutrosophic Crisp sets on a supra Neutrosophic Crisp topological space. In this research we used the notion of semi-a-closed Neutrosophic Crisp supra-sets to introduce new concepts, such as separation properties and new notions of sets. In addition, there are some existing relationships between them.

Keywords: Neutrosophic set theory; supra Neutrosophic crisp sets semi-a-closed; Neutrosophic separation axioms; supra topological space Neutrosophic Crisp; topological space

\section{Novo estudo dos conjuntos Supra Neutrosophic Crisp}

Resumo: tendo em vista que o mundo está cheio de indeterminação, os Neutrosophic encontraram seu lugar na pesquisa contemporânea. É por isso que, neste artigo, apresentamos um novo resultado de conjuntos Supra Neutrosophic Crisp sobre um espaço topológico Supra Neutrosophic Crisp. Nesta pesquisa, foi utilizada a noção de conjuntos Supra Neutrosophic Crisp semi-a-fechados para introduzir novos conceitos, como "propriedades de separação", e novas noções de conjuntos. Além disso, são encontradas algumas relações existentes entre elas.

Palavras-chave: teoria de conjuntos Neutrosophic; conjuntos Supra Neutrosophic Crisp semi-a-fechados; Neutrosophic axiomas de separação; espaço topológico Supra Neutrosophic Crisp; espaço topológico 


\section{Introducción}

El concepto de Neutrosophic es una nueva rama de la filosofía introducida por Smarandache [10], [12]. Este concepto tiene muchas aplicaciones en diferentes campos de las ciencias como, por ejemplo, la topología. Posteriormente, como una generalización del concepto de espacios topológicos, la noción de la teoría de conjuntos Neutrosophic Crisp fue estudiada inicialmente por Smarandache [11] y complementada por Salama et al. [7], [15]. Estos autores definieron y estudiaron propiedades sobre este tipo de conjuntos. Estos conjuntos fueron definidos como: sea $X$ un espacio muestral fijo no vacío, un conjunto Neutrosophic Crisp (NCS para abreviar), es un objeto a que tiene la forma $\mathrm{a}=<A_{1}$, $A_{2}, A_{3}>$, donde $A_{1}, A_{2}, A_{3}$ son subconjuntos de $X$, y son disyuntos uno a uno. Además, estos elementos son identificados o llamados un triplete ordenado. A partir de estas nociones, los topologistas empezaron a estudiar estos conjuntos, para lo cual tuvieron en cuenta otras nociones conocidas en la topología general, tales como conjuntos Neutrosophic Crisp preabiertos, conjuntos Neutrosophic Crisp semiabiertos y conjuntos Neutrosophic Crispa-abiertos [7]. Además, se han estudiado nuevas nociones combinando los conjuntos mencionados [2], como, por ejemplo, una clase de conjuntos Neutrosophic Crisp llamados conjuntos Neutrosophic Crisp semi alpha cerrados. Estos conjuntos utilizan las nociones de conjuntos Neutrosophic Crisp semiabiertos y Neutrosophic Crispa-abiertos a través de la clausura y el interior de dichos conjuntos.

Por otra parte, el concepto de Crisp supra espacio topológico fue introducido por [6] como una generalización del concepto de espacio topológico. Además, Jayaparthasarathy, Little y Arockia [5] generalizaron este concepto e introdujeron el concepto de espacio Neutrosophic supra topológico mediante el uso de conjuntos Neutrosophic fuzzy. Adicionalmente, R. Al-Hamido presentó un estudio más general, en el cual creó el concepto de espacios supra Neutrosophic Crisp topológicos [1]. Teniendo en cuenta lo mencionado, Al-Nafee y Al-Hamido [3], motivados por estos resultados introducen los conceptos de axiomas de separación sobre conjuntos Neutrosophic Crisp. Estas nociones estudiadas permitieron el desarrollo de los axiomas de separación sobre conjuntos supra Neutrosophic crisp que se estudian en las siguientes secciones de este artículo.

\section{Materiales y métodos}

Inicialmente, se realiza una documentación sobre las nociones de conjuntos supra Neutrosophic Crisp semi- $\alpha$-cerrados introducidas y estudiadas en [4] Posteriormente, se estudian algunas relaciones entre estos conjuntos y los conjuntos supra Neutrosophic Crisp $b-a-c e r r a d o s$ y supra Neutrosophic Crisp ${ }^{\star} b-a-c e r r a d o s$, introducidos en este artículo. Luego, se definen algunas propiedades de axiomas de separación sobre conjuntos supra Neutrosophic Crisp semi- $\alpha$-cerrados. Como validación de estos procesos se presentan las demostraciones de cada uno de ellos. Ahora bien, aquellas demostraciones que sean consecuencia de un teorema, una proposición, un lema o una definición anterior a esta se especificarán y no serán demostradas detalladamente; aquellos enunciados en los que su recíproco no se cumpla, se realizará un ejemplo. Finalmente, se establecen las conclusiones de los resultados y se proponen posibles futuros trabajos.

Iniciamos está sección planteando algunas definiciones básicas presentadas en [4].

Definición 1: una supra topología Neutrosophic crisp (NCST para abreviar) en un conjunto X no vacío, es una familia.

1. $\phi_{\mathrm{N}}, \mathrm{X}_{\mathrm{N}} \in \tau^{\mu}$

2. $\mathrm{E}_{\mathrm{i}} \in \tau^{\mu} \forall\left\{\mathrm{E}_{\mathrm{i}}: \mathrm{i} \in \mathrm{I}\right\} \subseteq \tau^{\mu}$

Entonces, el par $\left(\mathrm{X}, \tau^{\mu}\right)$ es llamado espacio topológico supra Neutrosophic crisp (NCSTS), los elementos $\tau^{\mu}$ son llamados conjuntos supra Neutrosophic crisp abiertos (NCsos) y el complemento de los elementos $\tau^{\mu}$ son llamados conjuntos supra Neutrosophic crisp supra cerrados (NCSCS). A través de este artículo, el par $\left(\mathrm{X}, \tau^{\mu}\right)$ será denotado por NCSTS.

Ejemplo 1: sea $X=\left\{\delta_{1}, \eta_{2}, \psi_{3}, \sigma_{4}\right\}, \tau^{\mu}=\left\{\phi_{N}, X_{N}, L\right.$, $\mathrm{M}, \mathrm{N}\}$, donde $\mathrm{L}=<\left\{\delta_{1}, \eta_{2}\right\}, \phi,\left\{\psi_{3}\right\}>,=<\left\{\delta_{1}, \eta_{2}\right\}, \phi$, $\left\{\sigma_{4}, \psi_{3}\right\}>, N=<\left\{\eta_{2}\right\}, \phi,\left\{\delta_{1}, \psi_{3}\right\}>$. Entonces $\left(X, \tau^{\mu}\right)$ es un espacio NCSTS. 
Definición 2: la supra Neutrosophic crisp interior y clausura de $E$ se define como:

1. $\operatorname{NCs}-c l(E)=\{\lambda: \lambda$ is a NCsCs in $X \& E \subseteq \lambda\}$.

2. NCS-int $(E)=\left\{\lambda^{*}: \lambda^{*}\right.$ is a NCsos in $\left.X \& \lambda^{*} \subseteq E\right\}$.

Definición 3: sea $\left(X, \tau^{\mu}\right)$ un espacio nCsTs y $E$ un subconjunto NC de $X$. Entonces $E$ se dice que es un conjunto:

1. Supra Neutrosophic Crisp pre-abierto (NCS-Pos para abreviar) si $E \subseteq$ NCs -int (NCS-cl (E)) y supra Neutrosophic Crisp supra pre-cerrado (NCS-P-CS para abreviar) si NCS-cl (NCS-int (E)) $\subseteq \mathrm{E}$.

2. Neutrosophic Crisp supra semi-open set (NCSP-Os para abreviar) si $E \subseteq$ NCs-cl (NCS-int (E )) \& Neutrosophic Crisp supra semi-cerrado (NCS-S-CS para abreviar) si NCS-int (NCS-cl (E)) $\subseteq \mathrm{E}$.

3. Neutrosophic Crisp supra- $\alpha$-abierto (NCS- $\alpha$-OS para abreviar) si $E \subseteq$ NCS-int (NCS-cl (NCS-int (E))) y Neutrosophic Crisp supra- $\alpha$-cerrado (NCS- $\alpha-$ CS para abreviar) si NCS-cl (NCS-int $(\mathrm{NCS}-\mathrm{cl}(\mathrm{E}))) \subseteq \mathrm{E}$.

4. Neutrosophic Crisp supra-semi pre-abierto (NCS-SP-OS para abreviar) si $E \subseteq$ NCS-cl (NCSint (NCS-Cl (E))) y Neutrosophic Crisp supra-semi pre-cerrado (NCS $\alpha$-CS para abreviar) si NCS-int (NCS-cl (NCS-int (E))) $\subseteq \mathrm{E}$.

Ejemplo 2: este ejemplo que muestra lo enunciado en la definición 3.

1. Sea $\left.\left.X=<\delta_{1}, \eta_{2}, \psi_{3}, \sigma_{4}\right\}, \tau^{\mu}=<\phi_{\mathrm{N}}, X_{N}, P, Q, R\right\}$, donde $P=<\left\{\delta_{1}, \eta\right\}, \phi,\left\{\psi_{3}\right\}>,=<\left\{\delta_{1}, \eta_{2}\right\}, \phi,\left\{\psi_{3}\right.$, $\left.\sigma_{4}\right\}>, R=<\left\{\eta_{2}\right\}, \phi,\left\{\delta_{1}, \psi_{3}\right\}>$, entonces $\left(X, \tau^{\mu}\right)$ es un espacio NCSTs si $E=\left\langle\left\{\eta_{2}\right\}, \phi,\left\{\delta_{1}, \psi_{3}\right\}>\right.$, así $E$ es un conjunto NCS-P-OS y $E^{C}=<\left\{\delta_{1}, \psi_{3}\right\}, \phi,\left\{\eta_{2}\right\}$ $>$ es un conjunto NCS-P-CS.

2. Sea $\left.\left.X=<\delta_{1}, \eta_{2}, \psi_{3}, \sigma_{4}\right\}, \tau^{\mu}=<\phi_{\mathrm{N}}, \mathrm{X}_{\mathrm{N}}, \mathrm{P}, \mathrm{Q}, \mathrm{R}\right\}$, donde $P=<\left\{\delta_{1}, \eta_{2}\right\},\left\{\psi_{3}\right\},\left\{\sigma_{4}\right\}>, Q=<\left\{\eta_{2}\right\}, \phi,\left\{\psi_{3}\right.$, $\left.\sigma_{4}\right\}>, R=<\left\{\eta_{2}\right\},\left\{\psi_{3}\right\},\left\{\delta_{1}, \sigma_{4}\right\}>$, entonces $\left(X, \tau^{\mu}\right)$ es un espacio NCsTs si $E=\left\langle\left\{\eta_{2}\right\}, \phi,\left\{\psi_{3}, \sigma_{4}\right\}\right\rangle$, así $E$ es un conjunto NCS-S-Os y $E^{\mathrm{C}}=<\left\{\psi_{3}, \sigma_{4}\right\}, \phi$, $\left\{\eta_{2}\right\}>$ es un conjunto NCS-S-CS.

3. Sea $\left.\left.X=<\delta_{1}, \eta_{2}, \psi_{3}, \sigma_{4}\right\}, \tau^{\mu}=<\phi_{\mathrm{N}}, \mathrm{X}_{\mathrm{N}}, \mathrm{P}, \mathrm{Q}, \mathrm{R}\right\}$, donde $P=<\left\{\delta_{1}, \eta_{2}\right\},\left\{\psi_{3}\right\},\left\{\sigma_{4}\right\}>, Q=<\left\{\eta_{2}\right\}, \phi,\left\{\psi_{3}\right.$, $\left.\sigma_{4}\right\}>, R=<\left\{\eta_{2}\right\},\left\{\psi_{3}\right\},\left\{\delta_{1}, \sigma_{4}\right\}>$, entonces $\left(X, \tau^{\mu}\right)$ es un espacio NCSTs si $E=\left\langle\left\{\delta_{1}, \eta_{2}\right\},\left\{\psi_{3}\right\},\left\{\sigma_{4}\right\}\right\rangle$, así $E$ es un conjunto NCS- $\alpha$-Os y $E^{C}=<\left\{\sigma_{4}\right\},\left\{\psi_{3}\right\}$, $\left\{\delta_{1}, \eta_{2}\right\}>$ es un conjunto NCS- $\alpha$-Cs.

4. Sea $\left.\left.X=<\delta_{1}, \eta_{2}, \psi_{3}, \sigma_{4}\right\}, \tau^{\mu}=<\phi_{N}, X_{N}, P, Q, R\right\}$, donde $P=<\left\{\delta_{1}, \eta_{2}\right\}, \phi,\left\{\psi_{3}\right\}>,=<\left\{\delta_{1}, \eta_{2}\right\}, \phi,\left\{\psi_{3}\right.$, $\left.\sigma_{4}\right\}>, R=<\left\{\eta_{2}\right\}, \phi,\left\{\delta_{1}, \psi_{3}\right\}>$, entonces $\left(X, \tau^{\mu}\right)$ es un espacio NCSTs si $E=\left\langle\left\{\eta_{2}\right\}, \phi,\left\{\psi_{3}, \sigma_{4}\right\}>\right.$, así $E$ es un conjunto NCS-SP-OS y $E^{C}=\left\langle\left\{\psi_{3}, \sigma_{4}\right\}, \phi\right.$, $\left\{\eta_{2}\right\}>$ es un conjunto NCS-SP-CS.

\section{Teorema 1:}

1. Todo conjunto NCSCs (NCSOS) es un conjunto NCS- $\alpha-$ CS (NCS- $\alpha$-OS).

2. Todo conjunto NCS- $\alpha$-CS (NCS- $\alpha-O S)$ es un conjunto NCS-P-CS (NCS-P-OS).

3. Todo conjunto NCS-S-CS (NCS-S-Os) es un conjunto NCS SP-CS (NCS-SP-OS).

\section{Demostración:}

1. Sea $E$ un conjunto NCSCS de $\left(\mathrm{X}, \tau^{\mu}\right) \Rightarrow$ NCS-cl $(\mathrm{E})=E$. Dado que NCs-cl $(\mathrm{E})=E$, se tiene que NCS-int $($ NCS-cl $(E))=$ NCS-int $(E) \subseteq \mathrm{E} \Rightarrow$ NCS$\mathrm{cl}($ NCS-int $($ NCS-cl (E))) $\subseteq$ NCS-cl (E) $\Rightarrow$ NCS-cl $(\mathrm{NCS}$-int $(\mathrm{NCS}-\mathrm{cl}(\mathrm{E}))) \subseteq$ E. Por lo tanto, E es un conjunto NCS- $\alpha-$ Cs de $\left(X, \tau^{\mu}\right)$.

2. Sea $E$ un conjunto NCS- $\alpha-$ CS de $\left(X, \tau^{\mu}\right) \Rightarrow$ NCS$\mathrm{cl}($ NCS-int $(\mathrm{NCS}-\mathrm{cl}(\mathrm{E}))) \subseteq \mathrm{E}$. Dado que NCs-int $(\mathrm{E}) \subseteq \mathrm{E} \subseteq \mathrm{NCs}-\mathrm{cl}(\mathrm{E})$, se tiene que NCs-int $(\mathrm{E}) \subseteq$ NCs-cl $(\mathrm{E}) \Rightarrow$ NCs-int $(\mathrm{E}) \subseteq$ NCs-int(NCS-cl (E)) $\Rightarrow$ NCS-cl(nCs-int (E)) $\subseteq$ NCs-cl(nCs-int(NCS-cl $(\mathrm{E}))) \subseteq \mathrm{E} \Rightarrow \operatorname{NCs}-\mathrm{cl}($ NCs-int $(\mathrm{E})) \subseteq \mathrm{E}$. Por lo tanto $E$ es un conjunto NCS-P-CS.

3. Sea $\mathrm{E}$ un conjunto NCS-S-CS de $\left(\mathrm{X}, \tau^{\mu}\right) \Rightarrow$ NCSint $($ NCs-cl $(\mathrm{E})) \subseteq \mathrm{E}$. Since NCs-int $(\mathrm{E}) \subseteq \mathrm{E} \subseteq$ NCs-cl (E), se tiene que NCs-int $(\mathrm{E}) \subseteq$ NCs-cl (E) $\Rightarrow$ NCS-cl(NCS-int(E)) $\subseteq$ NCS-cl(E) $\Rightarrow$ NCS-in$\mathrm{t}(\mathrm{NCS}-\mathrm{cl}(\mathrm{NCS}-\mathrm{int}(\mathrm{E}))) \subseteq \mathrm{NCS}-\mathrm{int}(\mathrm{NCS}-\mathrm{cl}(\mathrm{E})) \subseteq \mathrm{E}$ $\Rightarrow$ NCS-int(NCS-cl(NCS-int(E)) $\subseteq$ E. Por lo tanto, $E$ es un conjunto NCS-SP-CS.

Observación 1: los recíprocos del teorema 1, no se cumplen generalmente como se puede ver en el ejemplo 3.

Ejemplo 3:

1. Sea $\left.\left.X=<\delta_{1}, \eta_{2}, \psi_{3}, \sigma_{4}\right\}, \tau^{\mu}=<\phi_{N}, X_{N}, P, Q, R\right\}$, donde $\mathrm{P}=<\left\{\delta_{1}, \eta_{2}\right\},\left\{\psi_{3}\right\},\left\{\sigma_{4}\right\}>, \mathrm{Q}=<\left\{\eta_{2}\right\}, \phi$, $\left\{\psi_{3}, \sigma_{4}\right\}>, R=<\left\{\eta_{2}\right\},\left\{\psi_{3}\right\},\left\{\delta_{1}, \sigma_{4}\right\}>$, entonces $(X$, $\left.\tau^{\mu}\right)$ es un espacio NCSTS si $E=<\left\{\eta_{2}\right\},\left\{\psi_{3}\right\},\left\{\sigma_{4}\right\}$ 
$>$, así se tiene que E es un conjunto NCS- $\alpha-O S$, pero no es un conjunto NCSOS y $\mathrm{E}^{\mathrm{C}}=<\left\{\sigma_{4}\right\},\left\{\psi_{3}\right\}$, $\left\{\eta_{2}\right\}>\varepsilon \sigma$ un conjunto NCS- $\alpha-C S$, pero no es un conjunto NCSCS.

2. Sea $\left.\left.X=<\delta_{1}, \eta_{2}, \psi_{3}, \sigma_{4}\right\}, \tau^{\mu}=<\phi_{\mathrm{N}}, X_{N}, P, Q, R\right\}$, donde $\mathrm{P}=<\left\{\delta_{1}, \eta_{2}\right\}, \phi,\left\{\psi_{3}\right\}>, \mathrm{Q}=<\left\{\delta_{1}, \eta_{2}\right\}, \phi,\left\{\psi_{3}\right.$, $\left.\sigma_{4}\right\}>, R=<\left\{\eta_{2}\right\}, \phi,\left\{\delta_{1}, \psi_{3}\right\}>$, entonces $\left(X, \tau^{\mu}\right)$ es un espacio NCsTs si $\mathrm{E}=<\left\{\eta_{2}\right\}, \phi,\left\{\psi_{3}, \sigma_{4}\right\}>$, así se tiene que $E$ es un conjunto NCS-P-OS, pero no es un conjunto NCS-A-OS y E $\mathrm{E}^{\mathrm{C}}=\left\langle\left\{\psi_{3}, \sigma_{4}\right\}, \phi,\left\{\eta_{2}\right\}>\right.$ es un conjunto NCS-P-CS, pero no es un conjunto NCS-A-CS.

3. Sea $\left.\left.X=<\delta_{1}, \eta_{2}, \psi_{3}, \sigma_{4}\right\}, \tau^{\mu}=<\phi_{\mathrm{N}}, X_{N}, P, Q, R\right\}$, donde $\mathrm{P}=<\left\{\delta_{1}, \eta_{2}\right\}, \phi,\left\{\psi_{3}\right\}>, \mathrm{Q}=<\left\{\delta_{1}, \eta_{2}\right\}, \phi,\left\{\psi_{3}\right.$, $\left.\sigma_{4}\right\}>, R=<\left\{\eta_{2}\right\}, \phi,\left\{\delta_{1}, \psi_{3}\right\}>$, entonces $\left(X, \tau^{\mu}\right)$ es un espacio NCSTs si $\mathrm{E}=<\left\{\eta_{2}\right\}, \phi,\left\{\psi_{3}, \sigma_{4}\right\}>$, así se tiene que $E$ es un conjunto NCS-SP-OS, pero no es un CONJUnTo NCS-S-Os y $E^{C}=<\left\{\psi_{3}, \sigma_{4}\right\}, \phi$, $\left\{\eta_{2}\right\}>$ es un conjunto NCS-SP-CS, pero no es un conjunto NCS-S-CS.

\section{Resultados}

En esta sección se introduce y se estudian los conjuntos supra Neutrosophic Crisp semi-a-cerrados y su relación con algunos conjuntos, así como algunas propiedades de separación sobre estos conjuntos. Algunos de los resultados expuestos en esta sección fueron demostrados en [4], por lo que se presenta una generalización de los resultados obtenidos.

Definición 4: sea $\left(X, \tau^{\mu}\right)$ un espacio NCsTs y sea $E$ un subconjunto NC de $X$. Entonces se dice que $E$ es un conjunto supra Neutrosophic Crisp semi- $\alpha$-cerrado (NCS-p- $\alpha$-CS para abreviar) si existe un conjunto NCs- $\alpha$-CsH de $\left(X, \tau^{\mu}\right)$ tal que NCs-int $(\mathrm{H}) \subseteq \mathrm{E} \subseteq \mathrm{H}$. La familia de todos los conjuntos NCS-S- $\alpha$-CS, serán denotados por NCS-S- $\alpha-C s(X)$.

Definición 5: un conjunto Neutrosophic Crisp E se dice que es un conjunto supra neutrosophic crisp semi- $\alpha$-open (NCS-s $\alpha$-os para abreviar), si y solo si, $E^{C}$ es un conjunto NCS-sa-CS, es decir, si existe un conjunto NCS- $\alpha-O$ OS $\mathrm{He}\left(\mathrm{X}, \tau^{\mu}\right)$ tal que $\mathrm{H}$ $\subseteq \mathrm{E} \subseteq$ NCS-cl (H). La familia de todos los conjuntos NCS-Sa-OS, serán denotados por NCs-s $\alpha-O S(X)$.
Ejemplo 4: sea $\left.X=<\delta_{1}, \eta_{2}, \psi_{3}, \sigma_{4}\right\}, \tau^{\mu}=\left\{\phi_{\mathrm{N}}, X_{N}\right.$, $\mathrm{P}, \mathrm{Q}, \mathrm{R}\}$, donde $\mathrm{P}=<\left\{\delta_{1}, \eta_{2}\right\}, \phi,\left\{\psi_{3}\right\}>, \mathrm{Q}=<\left\{\delta_{1}\right.$, $\left.\eta_{2}\right\}, \phi,\left\{\sigma_{4}, \psi_{3}\right\}>, R=<\left\{\delta_{1}\right\}, \phi,\left\{\delta_{1}, \psi_{3}\right\}>$, entonces ( $\left.\mathrm{X}, \tau^{\mu}\right)$ es un espacio nCsTs. Ahora, Sea $\mathrm{H}=<\left\{\delta_{1}\right\}, \phi$, $\left\{\sigma_{4}\right\}>$ y $\mathrm{E}=<\phi, \phi,\left\{\delta_{1}, \sigma_{4}\right\}>$, así tenemos que E es un conjunto NCS-Sa-CS y $\mathrm{E}^{\mathrm{C}}=<\left\{\delta_{1}, \sigma_{4}\right\}, \phi, \phi>$ es un conjunto NCS-Sa-OS.

La siguiente proposición es seguida por la definición de $\left(X, \tau^{\mu}\right)$

\section{Proposición 1:}

1. Todo conjunto NCSCS (resp. NCSOS) es un conjunto NCS-Sa -CS (resp. NCS-Sa-OS).

2. Todo conjunto NCS- $\alpha$-CS (resp. NCS- $\alpha-$ OS) es un conjunto NCs-s $\alpha-\mathrm{CS}$ (resp. NCS-Sa-OS).

Observación 2: Los recíprocos de la proposición 4.4 no se cumplen generalmente como se puede ver en el ejemplo 4.6.

Ejemplo 5: Sea $\left.\mathrm{X}=<\delta_{1}, \eta_{2}, \psi_{3}, \sigma_{4}\right\}, \tau^{\mu}=\left\{\phi_{\mathrm{N}}, \mathrm{X}_{\mathrm{N}}\right.$, $\mathrm{P}, \mathrm{Q}, \mathrm{R}\}$, donde $\mathrm{P}=<\left\{\delta_{1}, \eta_{2}\right\}, \phi,\left\{\psi_{3}\right\}>, \mathrm{Q}=<\left\{\delta_{1}, \eta_{2}\right\}$, $\phi,\left\{\sigma_{4}, \psi_{3}\right\}>, \mathrm{R}=<\left\{\delta_{1}\right\}, \phi,\left\{\delta_{1}, \psi_{3}\right\}>$, entonces $\left(\mathrm{X}, \tau^{\mu}\right)$ es un espacio NCSTS. Ahora, sea $\mathrm{H}=\left\langle\left\{\delta_{1}\right\}, \phi,\left\{\sigma_{4}\right\}>\right.$ $\& \mathrm{E}=\left\langle\phi, \phi,\left\{\delta_{1}, \sigma_{4}\right\}>\right.$. Por lo tanto $\mathrm{E}$ es un conjunto NCs-S $\alpha$-CS, pero no es un conjunto NCSCS, $\mathrm{y} \mathrm{E}^{\mathrm{C}}=<$ $\left\{\delta_{1}, \sigma_{4}\right\}, \phi, \phi>$ es un conjunto NCS- $\alpha$-OS, pero no es un conjunto NCsos.

Proposición 2: sea $\left(X, \tau^{\mu}\right)$ un espacio NCSTS y sea $\mathrm{E}$ un conjunto $\mathrm{NC}$ de $\mathrm{X}, \mathrm{E} \in \mathrm{NCS}-\mathrm{a}-\mathrm{O}(\mathrm{X})$, si $\mathrm{y}$ solo si, a es un conjunto NCsos $\mathrm{H}$ tal que $\mathrm{H} \subseteq \mathrm{E} \subseteq$ NCS-int ( NCS-cl (H )).

Teorema 2: Sea $\left(X, \tau^{\mu}\right)$ un espacio NCSTS y sea $E$ un conjunto NC de X. Entonces, las siguientes propiedades son equivalentes:

1. $\mathrm{E} \in \mathrm{NCS}-\mathrm{S} \mathrm{a}-\mathrm{O}(\mathrm{X})$

2. Existe un conjunto NCsos, llamado $\mathrm{H}$, tal que $\mathrm{H}$ $\subseteq \mathrm{E} \subseteq \mathrm{NCS}-\mathrm{cl}(\mathrm{NCS}-\mathrm{int}(\mathrm{NCS}-\mathrm{cl}(\mathrm{H})))$.

3. E $\subseteq$ NCS-cl ( NCS-int ( NCS-cl ( NCS-int (E)))).

\section{Demostración:}

$\Rightarrow$ (b) Sea E $\in$ NCS-Sa-o(X). Entonces existe $\vartheta$ $\in$ NCs- $\alpha-O(X)$, tal que $\vartheta \subseteq \mathrm{E} \subseteq$ ( NCs-cl (9)). Esto implica que, existe un conjunto NCsos $\mathrm{H}$ tal que $\mathrm{H} \subseteq 9 \subseteq$ NCS-int ( NCS-cl (H )) (por la proposición 4.7), tenemos que NCS-cl (H ) $\subseteq$ NCs- $\chi \lambda(9) \subseteq$ NCScl (NCS-int (NCS-cl (H )), así NCS-cl (9) $\subseteq$ NCS-cl (NCS-int (NCS-Cl (H )). En consecuencia $\mathrm{H} \subseteq 9 \subseteq \mathrm{E}$ 
$\subseteq($ NCS-cl $(9)) \subseteq$ NCS-cl (NCS- int ( NCS-cl (H )). Por lo tanto $\mathrm{H} \subseteq \mathrm{E} \subseteq \mathrm{NCS}-\mathrm{cl}$ (NCS-int (NCS-cl $(\mathrm{H})$ ), para algún NCsos, $\mathrm{H}$.

$\Rightarrow$ (c) Suponga que existe un conjunto NCsos, llamado $\mathrm{H}$, tal que $\mathrm{H} \subseteq \mathrm{E} \subseteq \mathrm{NCS}-\mathrm{cl}$ (NCS -int (NCS$\mathrm{cl}(\mathrm{H})))$. En bien sabido que NCs-int $(\mathrm{E}) \subseteq \mathrm{E}$. Por otro lado, $\mathrm{H} \subseteq$ NCs-int (E) (dado que NCs-int (E) es el mayor conjunto NCsos contenido en $\mathrm{E}$ ). Se tiene que NCS-cl $(\mathrm{H}) \subseteq$ NCs-cl (NCS-int $(E))$, por lo tanto NCs-int (NCS-cl $(\mathrm{H})) \subseteq$ NCS-int (NCS-cl (NCSint (E))) , así NCs-cl (NCS-int (NCS-cl (H))) $\subseteq$ NCs$\mathrm{cl}($ NCS-int (NCS-cl (NCS-int (E)))). Pero, E $\subseteq$ NCS-cl (NCS-int (NCS-cl (H))) (Por hipótesis). Esto implica que $\mathrm{E} \subseteq \mathrm{NCS}-\mathrm{cl}(\mathrm{NCS}-\mathrm{int}(\mathrm{NCS}-\mathrm{cl}(\mathrm{H}))) \subseteq \mathrm{NCS}-\mathrm{cl}$ (NCS-int (NCS-cl (NCS-int (E)))), de esta manera se concluye que $\mathrm{E} \subseteq$ NCS-cl (NCS-int (NCS-cl (NCS-int (E)))).

(c) $\Rightarrow$ (a) Sea E $\subseteq$ NCS-cl (NCS int (NCS-cl (NCSint (E)))). Se debe probar que $\mathrm{E} \in \mathrm{NCS}-\mathrm{S} \alpha-\mathrm{O}(\mathrm{X})$. Since NCs-int (NCS-cl (NCS-int (E))) $\subseteq$ NCS-cl (NCS-int (E)). Así, NCS-cl (NCS-int (NCS-cl (NCS-int (E)))) $\subseteq$ NCS-cl (NCS-cl (NCS-int (E))) = NCS-cl (NCS-int $(\mathrm{E}$ )) but $\mathrm{E} \subseteq$ NCS-cl (NCS-int (NCS-cl (NCS-int (E)))) (por hipótesis). Por lo tanto, $\mathrm{E} \subseteq \mathrm{NCS}-\mathrm{cl}$ (NCS -int $($ NCs $-\mathrm{cl}($ NCs-int $(\mathrm{E})))) \subseteq$ NCs-cl $($ NCs-int $(\mathrm{E})) \Rightarrow \mathrm{E}$ $\subseteq$ NCS-cl (NCS-int (E)) . Ahora, sea $\vartheta=$ NCs-int (E). Por lo tanto, existe NCsos $\vartheta$ tal que $\vartheta \subseteq \mathrm{E} \subseteq$ NCS-cl (E). Por otro lado, $\vartheta$ es un conjunto NCs- $\alpha$-abierto (dado que 9 es un conjunto NCsos). Esto prueba que $\mathrm{E} \in \mathrm{NCS}-\mathrm{S} \alpha-\mathrm{O}(\mathrm{X})$.

Teorema 3: sea $\left(X, \tau^{\mu}\right)$ un conjunto NCSTs y sea $E$ un conjunto NC de X. Entonces, las siguientes propiedades son equivalentes.

1. $\mathrm{E} \in \mathrm{NCS}-\mathrm{s} \alpha \mathrm{C}(\mathrm{X})$.

2. Existe un conjunto NCsCs, llamado $G$, tal que NCS-int ( NCS-cl (NCS-int $(\mathrm{G}))) \subseteq \mathrm{E} \subseteq \mathrm{G}$.

3. $\operatorname{NCS}-i n t(\operatorname{NCS}-\mathrm{Cl}(\operatorname{NCS}-i n t(\operatorname{NCS}-\mathrm{cl}(\mathrm{E})))) \subseteq \mathrm{E}$.

\section{Demostración:}

a) $\Rightarrow$ (b) Dado que $E \in$ NCS-Sa -C(X), se tiene que $\mathrm{E}^{\mathrm{C}} \in \mathrm{NCs}-\mathrm{S} a-\mathrm{O}(\mathrm{X})$. Por tanto, existe un conjunto NCsos, $\mathrm{H}$ tal que $\mathrm{H} \subseteq \mathrm{E}^{\mathrm{C}} \subseteq \mathrm{NCS}$-cl ( NCs-int ( NCS-cl (H ))) (Por el teorema 4.8). Por tanto, ( NCS-cl $(\operatorname{NCS}-i n t(\operatorname{NCS}-c l(H)))^{\mathrm{C}} \subseteq\left(\mathrm{E}^{\mathrm{C}}\right)^{\mathrm{C}} \subseteq \mathrm{H}^{\mathrm{C}}$, i.e. $\operatorname{NCS}$-int (NCS-cl ( NCS-int $\left.\left.\left(\mathrm{H}^{\mathrm{C}}\right)\right)\right) \subseteq \mathrm{E} \subseteq \mathrm{H}^{\mathrm{C}}$. Ahora, sea $\mathrm{H}^{\mathrm{C}}=\mathrm{G}$, donde $\mathrm{G}$ es un conjunto NCSCS de X. Entonces NCS-int ( NCs-cl ( NCs-int $(G))) \subseteq \mathrm{E}$ $\subseteq \mathrm{G}$, para algún NCscs $\mathrm{G}$.

b) $\Rightarrow$ (c) Suponga que existe un conjunto NCSCs, llamado G, tal que G NCs-int ( NCs-cl ( NCs-int $(\mathrm{G}))) \subseteq \mathrm{E} \subseteq \mathrm{G}$, pero NCs-cl (E) es el más pequeño conjunto cerrado de E. Por tanto, NCs-cl (E) $\subseteq$ G, así NCs-int (NCS-cl $(E)) \subseteq$ NCs-int $(G) \Rightarrow$ NCScl (NCS-int (NCS-cl (E))) $\subseteq$ NCS-cl (NCS-int $(\mathrm{G})) \Rightarrow$ NCs-int (NCS-cl (NCs-int (NCS-cl (E)))) $\subseteq$ NCs-int $($ NCS-cl (NCS-int $(\mathrm{G}))) \subseteq \mathrm{E} \Rightarrow$ NCS-int ( NCS-cl ( NCS$\operatorname{int}(\operatorname{NCS}-\mathrm{cl}(\mathrm{E}))))$ Í E.

(c) $\Rightarrow$ (a) Sea NCS-int ( NCS-cl ( NCS-int ( NCS-cl $(\mathrm{E})))) \subseteq \mathrm{E}$. Se debe probar que $\mathrm{E} \in \mathrm{NCS}-\mathrm{S} \alpha-\mathrm{C}(\mathrm{X})$, i.e., probar que $\mathrm{E}^{\mathrm{C}} \in \mathrm{NCS}-\mathrm{S} \alpha-\mathrm{O}(\mathrm{X})$. Sea $\mathrm{E}^{\mathrm{C}} \subseteq$ ( NCS-int $(\text { NCS-cl }(\text { NCS-int }(\text { NCS-cl }(E))))^{\mathrm{C}}=$ NCS-Cl $($ NCS-int (NCS-Cl (NCS-int $\left.\left.\left.\left(\mathrm{E}^{\mathrm{C}}\right)\right)\right)\right)$. Hence $\mathrm{E}^{\mathrm{C}} \subseteq$ NCS-cl(NCS -int (NCS-cl (NCS-int $\left.\left.\left(\mathrm{E}^{\mathrm{C}}\right)\right)\right)$ ). Por tanto, esto prueba que $\mathrm{E}^{\mathrm{C}} \in \mathrm{NCS}-\mathrm{S} \alpha-\mathrm{O}(\mathrm{X}) \Rightarrow \mathrm{E}$ ÎnCs-s $\alpha-\mathrm{C}(\mathrm{X})$.

A continuación, se muestran unas nociones interior y clausura de los conjuntos estudiados.

Definición 6: sea $\left(X, \tau^{\mu}\right)$ un conjunto NCSTs y sea $E$ un conjunto NC de $X$. Entonces, NCs-sa-clausura y NCS-p--interior se definen como:

1. NCS-Sa-cl $(E)=\{\Upsilon: \Upsilon$ es un conjunto NCS-Sa-CS de $X$ y $\mathrm{E} \subseteq \Upsilon\}$.

2. $\nu \chi \sigma-\sigma \alpha-\imath v \tau(E)=\left\{\Upsilon^{*}: \Upsilon^{*}\right.$ es un conjunto NCSsa-os de $\mathrm{X}$ y $\left.\Upsilon^{*} \subseteq \mathrm{E}\right\}$.

Proposición 3: sea $\left(X, \tau^{\mu}\right)$ un conjunto NCSTS y sea $E$ un conjunto NC de $X$ Entonces, los siguientes enunciados siempre se cumplen:

1. $\mathrm{NCS}-\mathrm{s} \alpha-\mathrm{cl}(\mathrm{E})=\mathrm{E}$, si y solo si, E es un conjunto NCS-Sa-CS.

2. NCs$-\mathrm{s} \alpha-\operatorname{int}(\mathrm{E})=\mathrm{E}$, si y solo si, E es un conjunto NCS-Sa-Os.

3. NCS-sa-cl (E) es el conjunto más pequeño NCSsa-cs que contiene a $E$.

4. NCS-Sa-int (E) es el conjunto más grande NCSsa-os contenido en $E$.

Demostración: la prueba de este ejercicio, es consecuencia de la definición de $\left(X, \tau^{\mu}\right)$ y la definición 6. 
Proposición 4: sea $\left(X, \tau^{\mu}\right)$ un conjunto NCSTS y sea $E$ un conjunto NC de $X$. Entonces, los siguientes enunciados se satisfacen:

1. NCS-sa-int $\left(\mathrm{X}_{\mathrm{N}}-\mathrm{E}\right)=\mathrm{X}_{\mathrm{N}}-(\mathrm{NCS}-\mathrm{cl}(\mathrm{E}))$.

2. NCS-s $\alpha-\mathrm{cl}\left(\mathrm{X}_{\mathrm{N}}-\mathrm{E}\right)=\mathrm{X}_{\mathrm{N}}-($ NCS-int $(\mathrm{E}))$.

Demostración:

1. Por definición NCS-Sa-cl $(E)=\{\Upsilon: \Upsilon$ es un conjunto NCS-sa-Cs de X y E $\subseteq \Upsilon\}, X_{N}-($ NCS-cl(E)) $=\mathrm{X}_{\mathrm{N}}-\{\Upsilon: \Upsilon$ es un conjunto NCS-s $\alpha-\mathrm{Cs}$ de $\mathrm{X}$ y E $\subseteq \Upsilon\}=\left\{\mathrm{X}_{\mathrm{N}}-\mathrm{Y}: \Upsilon\right.$ es un conjunto NCS-sa-Cs de $\mathrm{X}$ y $\mathrm{E} \subseteq \Upsilon\}=\{\mathrm{H}: \mathrm{H}$ es un conjunto NCs-sa-os de $\mathrm{X}$ y $\left.\mathrm{H} \subseteq \mathrm{X}_{\mathrm{N}}-\mathrm{E}\right\}=\mathrm{NCS}-\mathrm{s} \alpha-\operatorname{int}\left(\mathrm{X}_{\mathrm{N}}-\mathrm{E}\right)$.

2. La prueba es similar a I.

Teorema 4: sea $(X, \tau \mu)$ un espacio NCsTs, $E$ y $F$ son dos conjuntos NC de $X$. Entonces los siguientes enunciados se satisfacen:

1. NCS-s $\alpha-\operatorname{cl}\left(\phi_{\mathrm{N}}\right)=\phi_{\mathrm{N}}, \mathrm{NCS}-\mathrm{s} \alpha-\mathrm{cl}\left(\mathrm{X}_{\mathrm{N}}\right)=\mathrm{X}_{\mathrm{N}}$.

2. $\mathrm{E} \subseteq \mathrm{NCS}-\mathrm{S} \alpha-\mathrm{cl}(\mathrm{E})$

3. $\mathrm{E} \subseteq \mathrm{F} \Rightarrow \mathrm{NCS}-\mathrm{s} \alpha-\mathrm{cl}(\mathrm{E}) \subseteq \mathrm{NCS}-\mathrm{S}-\mathrm{cl}(\mathrm{F})$.

4. NCS-sa-cl (E F) $\subseteq$ NCS-s $\alpha-\mathrm{cl}(\mathrm{E})$ NCs-s $\alpha-\mathrm{cl}(\mathrm{F})$.

5. NCS-s $\alpha-\mathrm{cl}(\mathrm{E}) \mathrm{NCS}-\mathrm{s} \alpha-\mathrm{cl}(\mathrm{F}) \subseteq \mathrm{NCS}-\mathrm{S} \alpha-\mathrm{cl}(\mathrm{E} F)$.

6. NCS-s $\alpha-\mathrm{cl}($ NCS-s $\alpha-\mathrm{cl}(\mathrm{E}))=$ NCS-s $\alpha-\mathrm{cl}(\mathrm{E})$.

\section{Demostración:}

La demostración 1 y 2 son consecuencia de la definición de $\left(X, \tau^{\mu}\right)$ y la proposición 5.2.

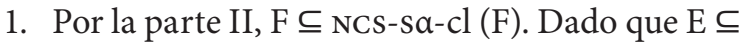
$\mathrm{F}$, se tiene que $\mathrm{E} \subseteq$ NCs-s $\alpha-\mathrm{cl}(\mathrm{F})$. Pero, NCs-s $\alpha-$ $\mathrm{cl}(\mathrm{F})$ es un conjunto NCs-s $\alpha-\mathrm{Cs}$. Así, NCS-s $\alpha-\mathrm{cl}$ (F) es un conjunto NCS-sa-Cs contenido en E. Dado que NCs-sa-cl (F) es el conjunto más pequeño NCs-sa-cs contenido en $E$, implica que NCs-s $\alpha-c l(E) \subseteq$ NCs-sa-cl (F) . Por lo tanto, E $\subseteq \mathrm{F} \Rightarrow$ NCS-S $\alpha-\mathrm{cl}(\mathrm{E}) \subseteq$ NCS-S $\alpha-\mathrm{cl}(\mathrm{F})$.

2. Se sabe que $\mathrm{E} F \subseteq \mathrm{E}$ y $\mathrm{E} F \subseteq \mathrm{F}$, por la parte (III) NCs-sa-cl (E F) $\subseteq$ NCs-sa-cl (E) NCs-sa-cl (E F) $\subseteq$ NCs-s $\alpha-\mathrm{cl}(\mathrm{F})$. Por lo tanto, NCS-s $\alpha-\mathrm{cl}(\mathrm{E} \mathrm{F}) \subseteq$, NCS-S $\alpha-\mathrm{cl}(\mathrm{E})$, NCS-s $\alpha-\mathrm{cl}(\mathrm{F})$.

1. Dado que $\mathrm{E} \subseteq \mathrm{E} F \mathrm{y} F \subseteq \mathrm{E}$, así se tiene que, NCS-S $\alpha-\mathrm{cl}(\mathrm{E}) \subseteq$, NCS-s $\alpha-\mathrm{cl}(\mathrm{E}$ F) y, NCS-S $\alpha$ $-\mathrm{cl}(\mathrm{F}) \subseteq$, NCS-sa-cl (E F). Por lo tanto, , NCSs $\alpha-\mathrm{cl}(\mathrm{E}), \mathrm{NCs}-\mathrm{s} \alpha-\mathrm{cl}(\mathrm{F}) \subseteq, \mathrm{NCS}-\mathrm{s} \alpha-\mathrm{cl}(\mathrm{E} F)$.

2. Dado que, NCs-sa-cl (E), esto implica que es un conjunto, NCS-s $\alpha-\mathrm{CS}$, ahora, por la proposición
5.2 parte (I), NCS-Sa-cl (, NCS-Sa-cl (E)) =, NCS$\mathrm{s} \alpha-\mathrm{cl}(\mathrm{E})$.

Teorema 5: sea $\left(X, \tau^{\mu}\right)$ un espacio NCsTs, $y$ sean $E$ y $F$ dos conjuntos NC de $X$. Entonces los siguientes enunciados se satisfacen:

1. NCs-s $\alpha$-int $\left(\phi_{\mathrm{N}}\right)=\phi_{\mathrm{N}}$, NCS-s $\alpha$ - int $\left(\mathrm{X}_{\mathrm{N}}\right)=\mathrm{X}_{\mathrm{N}}$.

2. NCS-sa-int $(\mathrm{E}) \subseteq \mathrm{E}$.

3. $\mathrm{E} \subseteq \mathrm{F} \Rightarrow$. NCS-sa-int $(\mathrm{E}) \subseteq \mathrm{NCS}$-Sa-int $(\mathrm{F})$.

4. NCS-sa-int $(E F) \subseteq$ NCS-s $\alpha$ - int (E) NCS-sa-int (F).

5. NCS-Sa-int (E) NCS-s $\alpha$ - int $(F) \subseteq$ NCs-s $\alpha$-int (E F).

6. NCS-Sa-int (NCS-Sa-int (E)) = NCS-Sa-int (E).

Demostración: la prueba de este teorema es similar a la del teorema 4.

Definición 7: sea $\left(\mathrm{X}, \tau^{\mu}\right)$ un espacio NCSTs y sea $E$ un subconjunto NC de $X$. Entonces se dice que $E$ es un conjunto supra neutrosophic crisp b-a-cerrado (NCs-b-a-Cs para abreviar) si existe un conjunto NCS-S- $\alpha-$ CS H de $\left(X, \tau^{\mu}\right)$ tal que NCs-s $\alpha$-int $(\mathrm{H}) \subseteq \mathrm{E} \subseteq \mathrm{H}$. La familia de todos los conjuntos NCs-b-a-Cs, serán denotados por NCs-b-a-Cs(X).

Proposición 5: todo conjunto supra Neutrosophic Crisp b-a-cerrado es un conjunto supra Neutrosophic Crisp S- $\alpha$-cerrado.

Demostración: es consecuencia de las definiciones 4,6 y 7 .

El siguiente ejemplo muestra que el recíproco de la proposición 5 no se cumple generalmente.

Ejemplo 6: sea $\mathrm{X}=<\delta_{1}, \eta_{2}, \psi_{3}, \sigma_{4}>, \tau^{\mu}=\left\{\phi_{\mathrm{N}}, \mathrm{X}_{\mathrm{N}}\right.$, $\mathrm{P}, \mathrm{Q}, \mathrm{R}\}$, donde $P=<\{\delta 1, \eta 2\}, \phi,\{\psi 3\}>, \mathrm{Q}=<\{\delta 1$, $\eta 2\}, \phi,\{\sigma 4, \psi 3\}>, R=<\{\delta 1\}, \phi,\{\delta 1, \psi 3\}>$, entonces $\left(\mathrm{X}, \tau^{\mu}\right)$ es un espacio ncsTs. Ahora, sea $\mathrm{H}=\left\langle\left\{\delta_{1}\right\}\right.$, $\phi,\left\{\sigma_{4}\right\}>$ y $\mathrm{E}=<\phi, \phi,\left\{\delta_{1}, \sigma_{4}\right\}>$, así tenemos que $\mathrm{E}$ es un conjunto NCS-Sa-CS, pero no es un conjunto NCs-b-a-cs.

Definición 8: sea $\left(\mathrm{X}, \tau^{\mu}\right)$ un espacio NCSTS y sea $E$ un subconjunto NC de X. Entonces, se dice que $E$ es un conjunto supra Neutrosophic Crisp ${ }^{*} b-a$-cerrado (NCS- ${ }^{*} b-a$-CS para abreviar) si existe un conjunto NCs-s- $\alpha-$ Cs $\mathrm{H}$ de $\left(\mathrm{X}, \tau^{\mu}\right)$ tal que NCssa-int (NCs-sa-cl $(\mathrm{H})) \subseteq \mathrm{E} \subseteq \mathrm{H}$. La familia de todos los conjuntos NCs- ${ }^{*} b-\alpha-C S$, serán denotados por NCs- ${ }^{*} b-a-C s(X)$. 
Proposición 6: todo conjunto supra Neutrosophic Crisp S- $\alpha$-cerrado es un conjunto supra Neutrosophic Crisp ${ }^{*} b$ - $\alpha$-cerrado.

Demostración:

El siguiente ejemplo muestra que el recíproco de la proposición 6 no se cumple generalmente.

Ejemplo 7: Sea $X=<\delta_{1}, \eta_{2}, \psi_{3}, \sigma_{4}>, \tau^{\mu}=\left\{\phi_{N}, X\right.$ $\mathrm{N}, \mathrm{P}, \mathrm{Q}, \mathrm{R}\}$, donde $\mathrm{P}=<\left\{\delta_{1}, \eta_{2}\right\}, \phi,\left\{\psi_{3}\right\}>, \mathrm{Q}=<\left\{\delta_{1}\right.$ ,$\left.\eta_{2}\right\}, \phi,\left\{\sigma_{4}, \psi_{3}\right\}>, \mathrm{R}=<\left\{\delta_{1}\right\}, \phi,\left\{\delta_{1}, \psi_{3}\right\}>$, entonces ( $\left.\mathrm{X}, \tau^{\mu}\right)$ es un espacio nCsTs. Ahora, sea $\mathrm{H}=<\left\{\delta_{1}\right\}, \phi$, $\{\sigma\}>$ y $\mathrm{E}=<\phi, \phi,\left\{\delta_{1}, \sigma_{4}\right\},\left\{\psi_{3}\right\}>$, así tenemos que $E$ es un conjunto NCs- ${ }^{*} b-\alpha-C S$, pero no es un conjunto NCS-Sa-Cs.

Lema 1: todo conjunto supra Neutrosophic Crisp b-a-cerrado es un conjunto supra Neutrosophic Crisp ${ }^{*} b$ - $a$-cerrado.

Demostración: consecuencia de las proposiciones 5 y 6.

Comentario 1: hasta este punto, podemos inferir que los conjuntos supra Neutrosophic Crisp $S$ - $\alpha$-cerrado cumplen las mismas propiedades de la clausura e interior de la topología clásica. No obstante, cumplir estas propiedades no implica que su comportamiento sea el mismo, pues tengamos en cuenta que la teoría Neutrosophic estudia estos conjuntos en intervalos de $[0,1]$. Lo que se quiere resaltar es que, al ser diferentes campos de la topología, sus propiedades se continúan conservando. Por otra parte, al introducir los conjuntos conjunto supra Neutrosophic Crisp ${ }^{*} b-a$-cerrado y conjunto supra Neutrosophic Crisp $b-a-c e r r a d o$ se encontraron las relaciones que se presentan en la Figura 1.

Además, debido a que todo conjunto supra Neutrosophic Crisp b-a-cerrado es un conjunto supra Neutrosophic Crisp S- $\alpha$-cerrado, los conjuntos supra Neutrosophic Crisp b-a-cerrado deben cumplir las condiciones de los teoremas 4 y 5, y proposición 3, pero debe ser probado; esta demostración puede ser un tema de interés para futuras investigaciones, así como también estudiar algunas propiedades de estos conjuntos.

A continuación, se muestran algunas propiedades de separación sobre los supra Neutrosophic Crisp semi- $\alpha$-cerrados y se introduce la noción funciones Neutrosophic Crisp semi- $\alpha$-continuas.

Se inicia con las propiedades de separación sobre los conjuntos supra Neutrosophic Crisp S- $\alpha$-cerrado.

Definición 9: un espacio topológico supra $\mathrm{Neu}-$ trosophic Crisp $\left(X, \tau^{\mu}\right)$ se dice que es:

1. $\mathrm{N}_{1}-\tau^{\mu} \mathrm{O}$-espacio si $\forall \mathrm{x}_{\mathrm{N} 1} \neq \mathrm{y}_{\mathrm{N} 1} \in \mathcal{X} \exists$ un conjunto supra Neutrosophic Crisp S- $\alpha$-abierto, $\mathrm{G}$ en $\mathcal{X}$ conteniendo uno de ellos, pero no al otro.

2. $\mathrm{N}_{2}-\tau{ }^{\mu} \mathrm{O}$-espacio si $\forall \mathrm{x}_{\mathrm{N} 2} \neq \mathrm{y}_{\mathrm{N} 2} \in \mathcal{X} \exists$ un conjunto supra Neutrosophic Crisp S- $\alpha$-abierto, $\mathrm{G}$ en $\mathcal{X}$ conteniendo uno de ellos, pero no al otro.

3. $\mathrm{N}_{3}-\tau{ }^{\mu} \mathrm{O}$-espacio si $\forall \mathrm{x}_{\mathrm{N} 3} \neq \mathrm{y}_{\mathrm{N} 3} \in \mathcal{X} \exists$ un conjunto supra Neutrosophic Crisp S- $\alpha$-abierto, $\mathrm{G}$ en $\mathcal{X}$ conteniendo uno de ellos, pero no al otro.

4. $\mathrm{N}_{1}-\tau^{\mu}{ }_{1}$-espacio si $\forall \mathrm{x}_{\mathrm{N} 1} \neq \mathrm{y}_{\mathrm{N} 1} \in \mathcal{X} \exists$ un conjunto supra Neutrosophic Crisp S-a-abierto $G_{1}$ y $G_{2}$ en $\mathcal{X}$ tal que $\mathrm{x}_{\mathrm{N} 1} \in \mathrm{G}_{1}, \mathrm{y}_{\mathrm{N} 1} \notin \mathrm{G}_{2}$ y $_{\mathrm{N}_{\mathrm{N} 1}} \notin \mathrm{G}_{2}, \mathrm{y}_{\mathrm{N} 1} \in$ $\mathrm{G}_{2}$.

5. N2 - $\tau^{\mu_{1}}$-espacio si $\forall \mathrm{x}_{\mathrm{N} 2} \neq \mathrm{y}_{\mathrm{N} 2} \in \mathcal{X} \exists$ un conjunto supra Neutrosophic Crisp S-a-abierto $G_{1}$ y $\mathrm{G}_{2}$ en $\mathcal{X}$ tal que $\mathrm{x}_{\mathrm{N} 2} \in \mathrm{G}_{1}, \mathrm{y}_{\mathrm{N} 2} \notin \mathrm{G}_{2} \mathrm{yx}_{\mathrm{N} 2} \notin \mathrm{G}_{2}, \mathrm{y}_{\mathrm{N} 2}$ $\in \mathrm{G}_{2}$.

6. N3 - $\tau^{\mu} 1$-espacio si $\forall \mathrm{x}_{\mathrm{N} 3} \neq \mathrm{y}_{\mathrm{N} 3} \in \mathcal{X} \exists$ un conjunto supra Neutrosophic Crisp S-a-abierto $G_{1}$ y $G_{2}$ en $\mathcal{X}$ tal que $\mathrm{x}_{\mathrm{N} 3} \in \mathrm{G}_{1}, \mathrm{y}_{\mathrm{N} 3} \notin \mathrm{G}_{2}$ y $_{\mathrm{N} 3} \notin \mathrm{G}_{2}, \mathrm{y}_{\mathrm{N} 3} \in$ $\mathrm{G}_{2}$.

7. N1 - $\tau^{\mu} 2$-espacio si $\forall \mathrm{x}_{\mathrm{N} 1} \neq \mathrm{y}_{\mathrm{N} 1} \in \mathcal{X} \exists$ un conjunto supra Neutrosophic Crisp S- $\alpha$-abierto $G_{1} y$

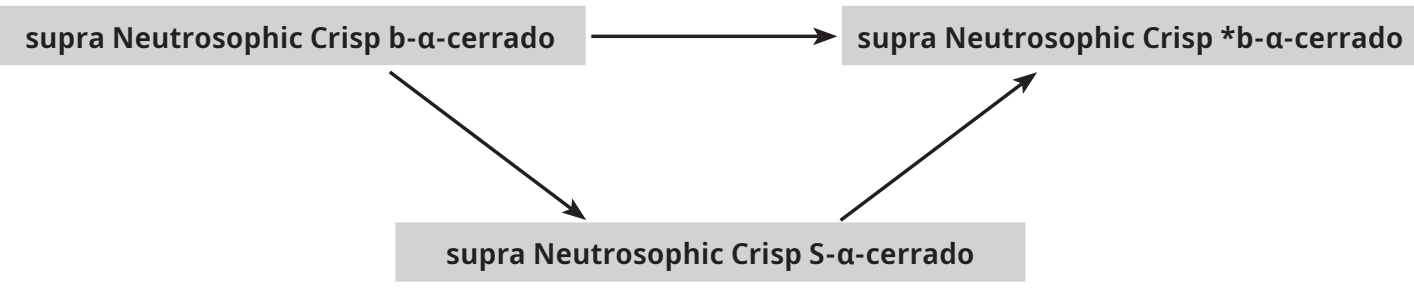

Figura 1. Relación entre los conjuntos.

Fuente: elaboración propia. 
$\mathrm{G}_{2}$ en $\mathcal{X}$ tal que $\mathrm{x}_{\mathrm{N} 1} \in \mathrm{G}_{\mathrm{N} 1}, \mathrm{y}_{\mathrm{N} 1} \notin \mathrm{G}_{1}$ y $_{\mathrm{N} 1} \notin \mathrm{G}_{2}, \mathrm{y}_{\mathrm{N} 1}$ $\in \mathrm{G}_{2}$ con $\mathrm{G}_{1} \cap \mathrm{G}_{2}=\varnothing$

8. N2 - $\tau^{\mu} 2$ - espacio si $\forall \mathrm{x}_{\mathrm{N} 2} \neq \mathrm{y}_{\mathrm{N} 2} \in \mathcal{X} \exists$ un conjunto supra Neutrosophic Crisp S- $\alpha$-abierto $\mathrm{G}_{1}$ y $\mathrm{G}_{2}$ en $\mathcal{X}$ tal que $\mathrm{x}_{\mathrm{N} 2} \in \mathrm{G}_{\mathrm{N} 2}, \mathrm{y}_{\mathrm{N} 2} \notin \mathrm{G}_{1}$ y $_{\mathrm{N} 2} \notin \mathrm{G}_{2}$, $\mathrm{y}_{\mathrm{N} 2} \in \mathrm{G}_{2} \operatorname{con} \mathrm{G}_{1} \cap \mathrm{G}_{2}=\varnothing$

9. N3 - $\tau^{\mu} 2$ - espacio si $\forall \mathrm{x}_{\mathrm{N} 3} \neq \mathrm{y}_{\mathrm{N} 3} \in \mathcal{X} \exists$ un conjunto supra Neutrosophic Crisp S- $\alpha$-abierto $G_{1}$ y $G_{2}$ en $\mathcal{X}$ tal que $\mathrm{x}_{\mathrm{N} 3} \in \mathrm{G}_{\mathrm{N} 3}, \mathrm{y}_{\mathrm{N} 3} \notin \mathrm{G}_{1} \mathrm{y} \mathrm{x}_{\mathrm{N} 3} \notin \mathrm{G}_{2}, \mathrm{y}_{\mathrm{N} 3} \in$ $\mathrm{G}_{2} \operatorname{con} \mathrm{G}_{1} \cap \mathrm{G}_{2}=\varnothing$

Definición 10: Un espacio topológico supra Neutrosophic Crisp (X, $\left.\tau^{\mu}\right)$ se dice que es:

1. $\mathrm{N}$ - $\tau^{\mu} \mathrm{O}$-espacio si $\left(\mathrm{X}, \tau^{\mu}\right)$ es un $\mathrm{N}_{1}-\mathcal{T}_{\mathrm{o}}$-espacio , $\mathrm{N}_{2}-\tau^{\mu}{ }_{0}$-espacio y $\mathrm{N}_{3}-\tau^{\mu}{ }_{0}$-espacio

2. $\mathrm{N}-\tau^{\mu}{ }_{1}$-espacio si $\left(\mathrm{X}, \tau^{\mu}\right)$ es un $\mathrm{N}_{1}-\mathcal{T}_{1}$-espacio, $\mathrm{N}_{2}-\tau^{\mu}{ }_{1}$-espacio y $\mathrm{N}_{3}-\tau^{\mu}{ }_{1}$-espacio

3. $\mathrm{N}-\tau^{\mu}{ }_{2}$-espacio si $\left(\mathrm{X}, \tau^{\mu}\right)$ es un $\mathrm{N}_{1}-\tau^{\mu}{ }_{2}$-espacio, $\mathrm{N}_{2}-\tau^{\mu}{ }_{2}$-espacio y $\mathrm{N}_{3}-\tau^{\mu}{ }_{2}$-espacio

Observación 2: para un espacio topológico supra Neutrosophic Crisp ( $\mathrm{X}, \tau^{\mu}$ ), las siguientes propiedades se cumplen:

1. Todo $\mathrm{N}$ - $\tau^{\mu} \mathrm{O}$-espacio es un $\mathrm{N} 1-\tau^{\mu} \mathrm{O}$-espacio.

2. Todo $\mathrm{N}-\tau^{\mu} 0$-espacio es un $\mathrm{N} 2-\tau^{\mu} 0$-espacio.

3. Todo $\mathrm{N}-\tau^{\mu} 0$-espacio es un $\mathrm{N} 3-\tau^{\mu} 0$-espacio.

Demostración: es consecuencia directa de las definiciones 9 y 10.

El siguiente ejemplo muestra que los recíprocos de la observación 2 no se cumplen generalmente.

Ejemplo 8: si $\mathrm{X}=<\delta_{1}, \eta_{2}>, \tau^{\mu} 1=\left\{\phi_{\mathrm{N}}, \mathrm{X}_{\mathrm{N}}, \mathrm{P}\right\}, \tau$ ${ }^{\mu} 2=\left\{\phi_{\mathrm{N}}, \mathrm{X}_{\mathrm{N}}, \mathrm{W}\right\}, \tau^{\mu} 3=\left\{\phi_{\mathrm{N}}, \mathrm{X}_{\mathrm{N}}, \mathrm{J}\right\}, \mathrm{P}=\left\langle\left\{\delta_{1}\right\}, \phi, \phi>\right.$ , $\mathrm{W}=<\phi,\left\{\eta_{2}\right\}, \phi>, J=<\phi, \phi,\left\{\delta_{1}\right\}>$, entonces $(\mathcal{X}, \tau$ $\left.{ }^{\mu} 1\right)$ es un $\mathrm{N} 1-\tau{ }^{\mu} \mathrm{O}$-espacio, pero no es un $\mathrm{N}-\tau{ }^{\mu} \mathrm{o}$-espacio, $\left(X, \tau^{\mu} 2\right)$ es un $\mathrm{N} 2-\tau^{\mu}$ o-espacio, pero no es un $\mathrm{N}-\tau^{\mu} \mathrm{O}$-espacio, $\left(\mathcal{X}, \tau^{\mu} 3\right)$ es un $\mathrm{N} 3-\tau^{\mu} \mathrm{O}$-espacio, pero no es un $\mathrm{N}-\tau^{\mu} \mathrm{O}$-espacio.

Observación 3: para un espacio topológico supra Neutrosophic Crisp $\left(X, \tau^{\mu}\right)$ las siguientes propiedades se cumplen:

1. Todo $\mathrm{N}-\tau^{\mu} 1$-espacio es un $\mathrm{N} 1-\tau{ }^{\mu} 1$-espacio.
2. Todo $\mathrm{N}-\tau^{\mu} 1$-espacio es un $\mathrm{N} 2-\tau^{\mu} 1$-espacio.

3. Todo $\mathrm{N}-\tau^{\mu} 1$-espacio es un $\mathrm{N} 3-\tau^{\mu} 1$-espacio.

Demostración: es consecuencia directa de las definiciones 9 y 10. Por otro lado, los recíprocos de la observación 3 no se cumplen generalmente, como se puede ver en el ejemplo 9.

Ejemplo 9: si $\mathrm{X}=<\delta_{1}, \eta_{2}>, \tau^{\mu} 1=\left\{\phi_{\mathrm{N}}, \mathrm{X}_{\mathrm{N}}, \mathrm{P}, \mathrm{Q}\right.$, $J\}, \tau^{\mu} 2=\left\{\phi_{\mathrm{N}}, \mathrm{X}_{\mathrm{N}}, \mathrm{Q}, \mathrm{X}, \mathrm{P}\right\}, \mathrm{P}=<\left\{\delta_{1}\right\},\left\{\eta_{2}\right\}, \phi>, \mathrm{Q}=<$ $\left\{\eta_{2}\right\},\left\{\delta_{1}\right\}, \phi>, J=<\left\{\delta_{1}\right\}, \phi, \phi>, X=<\left\{\eta_{2}\right\}, \phi, \phi>$, entonces $\left(X, \tau^{\mu} 1\right)$ es un $\mathrm{N} 1-\tau^{\mu} 1$-espacio, pero no es un $\mathrm{N}-\tau^{\mu}{ }_{1}$-espacio. $\left(X, \tau^{\mu} 1\right)$ es un $\mathrm{N} 2-\tau^{\mu} 1$-espacio, pero no es un $\mathrm{N}-\tau^{\mu} 1$-espacio. $\left(\mathcal{X}, \tau^{\mu} 2\right)$ es un N3 $-\tau$ ${ }^{\mu} 1$-espacio, pero no es un $\mathrm{N}-\tau^{\mu} 1$-espacio.

Observación 4: para cualquier espacio supra topología Neutrosophic Crisp $\left(X, \tau^{\mu}\right)$ los siguientes enunciados se cumplen:

1. Todo $\mathrm{N}-\tau^{\mu}{ }^{2}$-espacio es un $\mathrm{N} 1-\tau^{\mu} 2$-espacio.

2. Todo $\mathrm{N}-\tau^{\mu}{ }^{\mu}$-espacio es un $\mathrm{N} 2-\tau^{\mu}{ }^{2}$-espacio.

3. Todo $\mathrm{N}-\tau^{\mu}{ }^{2}$-espacio es un $\mathrm{N} 3-\tau^{\mu} 2$-espacio.

Demostración: es consecuencia directa de las definiciones 9 y 10. Por otro lado, los recíprocos de la observación 4 no se cumplen generalmente, como se puede ver en el ejemplo 9.

Observación 5: para cualquier espacio supra topología Neutrosophic Crisp $\left(\mathcal{X}, \tau^{\mu}\right)$, los siguientes enunciados se cumplen:

1. Todo $\mathrm{N}-\tau^{\mu} 1$-espacio es un $\mathrm{N}-\tau^{\mu} 0$-espacio.

2. Todo $\mathrm{N}-\tau^{\mu}{ }^{\mu}$-espacio es un $\mathrm{N}-\tau^{\mu}{ }^{\mu} 1$-espacio.

Demostración: es consecuencia directa de las definiciones 9 y 10.

El siguiente ejemplo muestra que el recíproco de la observación 5 no se cumple generalmente.

Ejemplo 10: si $\mathrm{X}=<\delta_{1}, \eta_{2}>, \tau^{\mu} 1=\left\{\phi_{\mathrm{N}}, \mathrm{X}_{\mathrm{N}}, \mathrm{P}, \mathrm{Q}\right.$, $\mathrm{J}\}, \mathrm{P}=<\left\{\delta_{1}\right\}, \phi, \phi>, \mathrm{Q}=<\phi,\left\{\eta_{2}\right\}, \phi>, \mathrm{J}=<\phi, \phi,\left\{\delta_{1}\right\}$ $>$, entonces $\left(\mathcal{X}, \tau^{\mu}\right)$ es un $\mathrm{N}$-t ${ }^{\mathrm{m}} \mathrm{O}$-espacio, pero no es un $\mathrm{N}-\tau^{\mu} 1$-espacio.

Comentario 2: teniendo en cuenta lo planteado, se tienen las relaciones que se presentan en la Figura 2. 

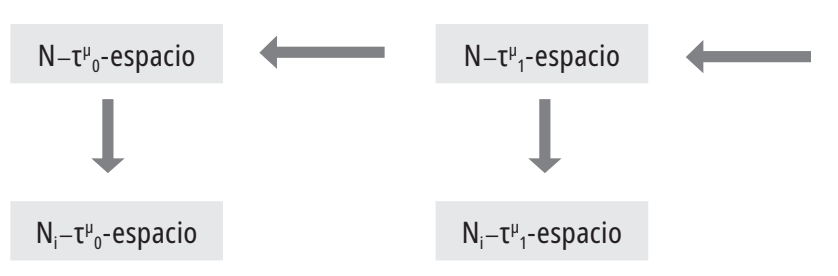

Figura 2. Relación entre los $\mathrm{N}$ - ${ }^{\mu}$-espacios.

Fuente: elaboración propia.

\section{Conclusión}

La idea principal de este artículo era mostrar algunos resultados obtenidos sobre los conjuntos los supra Neutrosophic Crisp semi- $\alpha$-cerrados, lo que es un complemento a lo presentado por [4]. Sobre estos conjuntos se probaron nuevas propiedades y se definieron algunos conjuntos, lo cual mostró la relación que habían entre ellos (véase la Figura 1). Por otra parte, se introduce el concepto de propiedades de separación sobre los conjuntos supra Neutrosophic Crisp semi- $\alpha$-cerrados y se establecen algunas relaciones existentes entre estos espacios (véase la Figura 2). Esta investigación constituye un aporte al estudio de los conjuntos supra Neutrosophic Crisp, introducido hace unos años. Para futuras investigaciones se recomienda estudiar las propiedades de los conjuntos supra Neutrosophic Crisp b- $a$-cerrados y supra Neutrosophic Crisp ${ }^{\star} b$ - $a$-cerrados. Además, se puede definir y estudiar el concepto de función sobre estos conjuntos que hasta el momento no han sido introducidos. Adicionalmente, esta noción puede ser estudiada y extendida en la topología neutrosofica refinada y, de esta manera, encontrar nuevas relaciones y propiedades.

\section{Conflictos de interés}

El autor manifiesta que no existe ningún conflicto de intereses en la publicación de la investigación.

\section{Referencias}

[1] R. Al-Hamido, "Neutrosophic Crisp Supra Bi-Topological Spaces", Int. J. Neutros. Sc., vo. 3, n. ${ }^{\circ} 3$, pp. 11-14, 2020. [En línea]. Disponible en: https://www.researchgate.net/publication/340915464_ON_Neutrosophic_ Crisp_Supra_Bi-Topological_Spaces
[2] R. Al-Hamido, Q. Imran, K. Alghurab y T. Gharibah, "On Neutrosophic Crisp Semi alpha closed sets", Neutros. Sets Sy., vol. 21, pp. 28-35, 2018. [En línea]. Recuperado de https://digitalrepository.unm.edu/cgi/ viewcontent.cgi?article $=1261 \&$ context $=$ nss_journal

[3] A. Al-Nafee, R. Al-Hamido y F. Smarandache, Separation axioms in neutrosophic crisp topological spaces. Neutrosophic sets and Systems, vol. 25, pp. 25-34, 2019. [En línea]. Recuperado de https://zenodo.org/ record/2631488\#.X5nBHYgzaUk

[4] V. Amarendra y P. Rajasekhar, On neutrosophic crisp supra semi-alpha closed sets, In. J. Adv. Sc. Tech., vol. 29, n. ${ }^{\circ}$ 6, pp. 2947-2954, 2020. Disponible en: http://sersc.org/journals/index.php/IJAST/article/view/13825

[5] G. Jayaparthasarathy, V. Little y M. Arockia, "Neutrosophic supra topological applications in data mining process", Neutros. Sets Sys., 27, pp. 80-97, 2019. Disponible en: https://www.researchgate.net/publication/334586721_Neutrosophic_Supra_Topological_Applications_in_Data_Mining_Process

[6] S. Mashhour,A. Allam, F. Mahmoud y F. Khedr, "On supratopological spaces", In. J. Pu. Appl. Math vol. 14, n. ${ }^{\circ}$ 4, pp. 502-510, 1983. [En línea]. Disponible en: https://insa.nic.in/writereaddata/UpLoadedFiles/ IJPAM/20005a7c_502.pdf

[7] A. Salama, Basic structure of some classes of neutrosophic crisp nearly open sets and possible. Neutros. Sets Sy., vol. 7, pp. 18-22, 2015. [En línea]. Disponible en: https://www.researchgate.net/publication/282442431_Basic_Structure_of_Some_Classes_of_Neutrosophic_Crisp_Nearly_Open_Sets_Possible_Application_to_GIS_Topology

[8] A. Salama y F. Smarandache, Neutrosophic crisp set theory. México: Educational Publisher Columbus, 2014. [En línea]. Disponible en: https://digitalrepository.unm.edu/cgi/viewcontent.cgi?article=1120\&context $=$ math_fsp

[9] A. Salama, F. Smarandache y V. Kroumov, Neutrosophic Crisp Sets and Neutrosophic Crisp Topological Spaces. Neutros. Sets Sys., vol. 2, pp. 25-30, 2014. [En 
línea]. Disponible en: http://fs.unm.edu/ScArt/NeutrosophicCrispSets.pdf

[10] F. Smarandache, Neutrosophy/Neutrosophic probability, set, and logic. México: American Research Press, 1998. [En línea]. Disponible en: https://www.researchgate.net/publication/305140002_Neutrosophic_Probability_Set_And_Logic_first_version

[11] F. Smarandache, A unifying field in logics: neutrosophic logic, neutrosophy, neutrosophic set, neutrosophic probability. Rehoboth: American Research Press, 1999. [En línea]. Disponible en: https://arxiv.org/ftp/math/ papers/0101/0101228.pdf
[12] F. Smarandache, Neutrosophy and Neutrosophic Logic, First International Conference on Neutrosophy, Neutrosophic Logic, Set, Probability, and Statistics. México, Gallup: University of New Mexico, 2001. [En línea]. Disponible en: https://www.researchgate.net/ publication/2107647_Proceedings_of_the_First_International_Conference_on_Neutrosophy_Neutrosophic_Logic_Neutrosophic_Set_Neutrosophic_Probability_and_Statistics 
